\title{
钯催化下稳定磷叶立德与烯丙基醇的脱水偶联反应
}

\author{
马献涛*, $a$ 于 静 ${ }^{a}$ 马瑞甜 ${ }^{a}$ 燕 然 ${ }^{a}$ 张振雷 $*, a, b$ \\ ( ${ }^{a}$ 信阳师范学院化学化工学院 信阳 464000) \\ ( ${ }^{b}$ 皁阳师范学院化学与材料工程学院 阜阳 236037)
}

\begin{abstract}
摘要 报道了钯催化下酮基稳定的磷叶立德与烯丙基醇一锅法的烯丙化-Wittig 反应. 研究表明, 在 $5 \mathrm{~mol} \%$ 四(三苯基 膦)钯和 $20 \mathrm{~mol} \%$ 硼酸的共催化下，以 $52 \% \sim 95 \%$ 的收率得到官能化 1,4-二烯化合物. 该方法还可以进一步拓展到酯基 以及氧基稳定的磷叶立德来合成对应的 $1,4-$ 二烯化合物.

关键词＼cjkstart稳定磷叶立德; 烯丙基醇; 脱水偶联; 钯催化
\end{abstract}

\section{Palladium-Catalyzed Dehydrative Cross Couplings of Stabilized Phosphorus Ylides with Allylic Alcohols}

\author{
Ma, Xiantao ${ }^{* a} \quad$ Yu, Jing ${ }^{a} \quad$ Ma, Ruitian ${ }^{a} \quad{\text { Yan, } \text { Ran }^{a} \quad \text { Zhang, Zhenlei }}^{*, a, b}$ \\ ( ${ }^{a}$ College of Chemistry and Chemical Engineering, Xinyang Normal University, Xinyang 464000) \\ ( ${ }^{b}$ School of Chemistry and Material Engineering, Fuyang Normal University, Fuyang 236037)
}

\begin{abstract}
A dehydrative cross coupling of ketone-stabilized phosphorus ylides with the readily available allylic alcohols followed by an one-pot Wittig reaction is developed. A range of functional 1,4-dienes could be obtained in $52 \% \sim 95 \%$ isolated yields in the presence of $5 \mathrm{~mol} \% \mathrm{Pd}\left(\mathrm{PPh}_{3}\right)_{4}$ and $20 \mathrm{~mol} \% \mathrm{~B}(\mathrm{OH})_{3}$. The same method can be extended to ester or nitrile-stabilized phosphorus ylides, affording the corresponding 1,4-dienes in moderate yields.

Keywords stabilized phosphorus ylides; allylic alcohols; dehydrative cross couplings; palladium catalysis
\end{abstract}

1,4-二烯结构广泛存在于天然产物及药物活性分子 中 $^{[1]}$. 目前, 文献中已有较多的 $1,4-$ 二烯的合成方法 ${ }^{[2 \sim 6]}$, 如过渡金属催化下烯基金属试剂的烯丙基偶联反 应 $^{[3]}$ 、烯/炔烃的官能化反应等 ${ }^{[4,5]}$. 磷叶立德是一种重要 的合成中间体, 被广泛应用于有机合成中. 如磷叶立德 与醛、酮的 Wittig 反应是制备取代烯烃的重要合成方 法 $^{[7]} .2010$ 年, 游书力课题组 ${ }^{[8]}$ 首次报道了钯催化下烯 丙基酯与酯基稳定磷叶立德的一锅法烯丙化-Wittig 反 应，用于酯基取代的 1,4-二烯化合物的合成(Scheme $1 \mathrm{~A}) ; 2013$ 年, 田仕凯课题组 ${ }^{[8]}$ 发展了烯丙基一级胺与 稳定磷叶立德的烯丙基偶联反应，用于酮基、酯基以及 氧基取代的 1,4-二烯化合物的合成(Scheme 1A). 与烯丙 基酯类化合物相比，烯丙基醇具有廉价易得，低毒稳定， 参与反应时离去基团小、原子经济性好等优点, 近年来
得到了合成化学家们的青睐 ${ }^{[9]}$. 最近, 我们课题组 ${ }^{[10]}$ 报 道了水相体系中烯丙基醇与稳定磷叶立德的脱水偶联 反应，然而该方法仅适用于酮基取代的 1,4-二烯化合物 的合成(Scheme 1B). 基于对醇的取代反应的兴趣 ${ }^{[11]}$ ，报 道了四(三苯基膦)钯催化下烯丙基醇与稳定磷叶立德的 一锅法脱水偶联反应，用于酮基、酯基以及氧基取代的 1,4-二烯化合物的高效合成(Scheme 1C).

\section{1 结果与讨论}

以肉桂醇 $(1 a)$ 与苯甲酰基稳定的磷叶立德 $(2 a)$ 作为 模板反应对反应条件进行了篮选. 根据已有经验, 反应 初始条件设定为: 肉桂醇 $(1 \mathbf{a}, 0.36 \mathrm{mmol}$ )、苯甲酰基稳定 的磷叶立德 $(2 \mathbf{a}, 0.30 \mathrm{mmol}) 、 \mathrm{Pd}\left(\mathrm{PPh}_{3}\right)_{4}(5 \mathrm{~mol} \%)$ 、硼酸 $(20 \mathrm{~mol} \%)$ 、乙腈 $(0.50 \mathrm{~mL})$, 氮气保护下置于 $110{ }^{\circ} \mathrm{C}$ 加

* Corresponding authors. E-mail: xiantaoma@126.com; helenken@mail.ustc.edu.cn

Received December 31, 2018; revised January 27, 2019; published online February 22, 2019.

Project supported by the Scientific Research Project of Henan Province (No. 19B150018), the Nanhu Scholars Program for Young Scholars of Xinyang Normal University and the Young Core Instructor Program of Xinyang Normal University (No. 2018GGJS-05).

河南省高等学校重点科研项目(No. 19B150018)、信阳师范学院 “南湖学者奖励计划” 青年项目和信阳师范学院青年骨干教师资助计划(No. 2018GGJS-05)资助项目. 
(A) Previous work reported by You's and Tian group

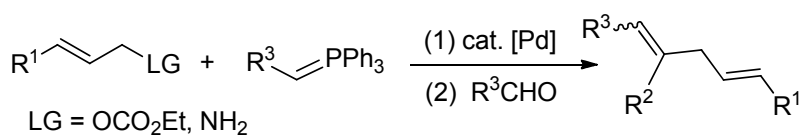

(B) our previous work

(1) $[\mathrm{Pd} \text { (allyl)Cl }]_{2}(2.5 \mathrm{~mol} \%)$

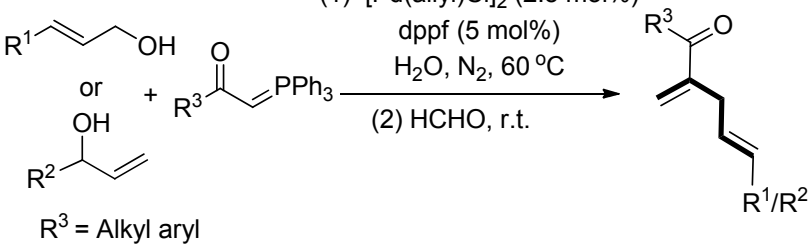

(C) This work: dehydrative cross coupling of allylic alcohols

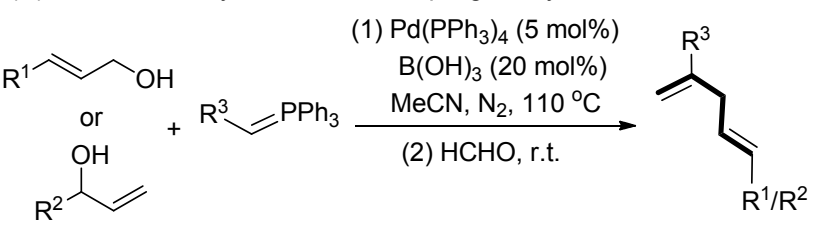

$\mathrm{R}^{3}=\mathrm{RCO}, \mathrm{EtOCO}, \mathrm{CN}$

图式 1 稳定磷叶立德的烯丙基偶联反应

Scheme 1 Catalytic cross coupling of stabilized phosphorus ylides with allylic partners

热搅拌反应 $12 \mathrm{~h}$; 待反应液冷却至室温后, 加入甲醛水 溶液(3 equiv.), 室温下继续搅拌 $6 \mathrm{~h}$, 以 $84 \%$ 的分离收率 得到预期的 1,4-二烯产物 3a(表 1, Entry 1). 对照实验表 明, 反应在没有钯催化剂或添加剂的条件下, 均不能顺 利进行, 说明钯催化剂和添加剂在活化烯丙基醇 $\mathrm{C}-\mathrm{O}$ 键断裂过程中起着至关重要的作用(Entries 2, 3). 随后, 对反应的添加剂进行了䇻选, 结果发现使用其他的添加 剂时, 并不能得到更好的反应收率(Entries 4, 5), 因此, 选定嗍酸作为最优添加剂. 对反应溶剂的篮选发现, 乙 腈是最优的反应溶剂(Entries 6 9). 对反应温度筷选发 现, 降低温度至 $60{ }^{\circ} \mathrm{C}$, 只能观测到少量产物的生成 (Entry 10).

将以上优化的反应条件(表 1 , Entry 1)用于各种取代 的烯丙基醇与酮基稳定磷叶立德的偶联反应, 结果如表 2 所示. 对于取代的肉桂醇, 当苯环上带有供电子基时, 可以顺利地与磷叶立德 $(\mathbf{2 a})$ 发生反应, 更加高效地得到 官能化 1,4-二烯产物 3b (表 2, Entries 1, 2). 与之相反, 当苯环上带有强吸电子基, 如硝基时, 可能是不利于 $\pi$ 烯丙基钯正离子中间体的稳定存在，只能观测到少量产 物 3c 生成(Entry 3). 当苯环含有邻位位阻的取代基时, 仍可以高效地得到官能化 1,4-二烯产物 3d (Entry 4). 该 方法也适用于脂肪型取代的烯丙基醇, 如(E)-2-己烯-1醇, 以单一的区域选择性中等收率得到预期产物 $3 \mathrm{e}$ (Entry 5). 对于简单的烯丙基醇, 也可以得到预期产物 3f (Entry 6). 对于 $\alpha$-取代的烯丙基二级醇, 可以顺利地 发生反应, 专一选择性地得到直链型的烯丙基偶联产
表 1 钯催化下肉桂醇与苯甲酰基稳定磷叶立德烯丙化反应 的条件篮选 ${ }^{a}$

Table 1 Condition screening for Pd-catalyzed allylic cross coupling of phosphorus ylide 2a with cinnamyl alcohol 1a

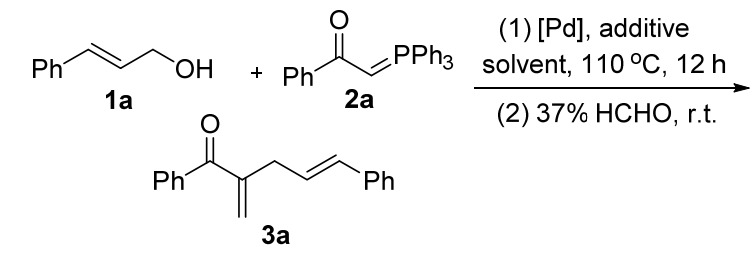

\begin{tabular}{ccclc}
\hline Entry & {$[\mathrm{Pd}]$} & Additive & Solvent & Yield $^{b} \%$ \\
\hline 1 & $\mathrm{Pd}\left(\mathrm{PPh}_{3}\right)_{4}$ & $\mathrm{~B}(\mathrm{OH})_{3}$ & $\mathrm{MeCN}$ & 84 \\
2 & $\mathrm{Pd}\left(\mathrm{PPh}_{3}\right)_{4}$ & - & $\mathrm{MeCN}$ & 0 \\
3 & - & $\mathrm{B}(\mathrm{OH})_{3}$ & $\mathrm{MeCN}$ & 0 \\
4 & $\mathrm{Pd}\left(\mathrm{PPh}_{3}\right)_{4}$ & $\mathrm{ZnCl}$ & $\mathrm{MeCN}$ & 32 \\
5 & $\mathrm{Pd}\left(\mathrm{PPh}_{3}\right)_{4}$ & $\mathrm{TsOH}$ & MeCN & 51 \\
6 & $\mathrm{Pd}\left(\mathrm{PPh}_{3}\right)_{4}$ & $\mathrm{~B}(\mathrm{OH})_{3}$ & DMSO & 28 \\
7 & $\mathrm{Pd}\left(\mathrm{PPh}_{3}\right)_{4}$ & $\mathrm{~B}(\mathrm{OH})_{3}$ & DMF & 82 \\
8 & $\mathrm{Pd}\left(\mathrm{PPh}_{3}\right)_{4}$ & $\mathrm{~B}(\mathrm{OH})_{3}$ & Toluene & 80 \\
9 & $\mathrm{Pd}\left(\mathrm{PPh}_{3}\right)_{4}$ & $\mathrm{~B}(\mathrm{OH})_{3}$ & Dioxane & 75 \\
$10^{c}$ & $\mathrm{Pd}\left(\mathrm{PPh}_{3}\right)_{4}$ & $\mathrm{~B}(\mathrm{OH})_{3}$ & MeCN & Trace \\
\hline
\end{tabular}

${ }^{a}$ Unless otherwise noted, the mixture of 1a $(0.36 \mathrm{mmol}, 1.2$ equiv. $), 2 \mathrm{a}(0.30$ $\mathrm{mmol})$, Pd catalyst $(5 \mathrm{~mol} \%)$, additive $(20 \mathrm{~mol} \%)$ and solvent $(0.5 \mathrm{~mL})$ was sealed under $\mathrm{N}_{2}$, heated at $110{ }^{\circ} \mathrm{C}$ for $12 \mathrm{~h}$, then $\mathrm{HCHO}(37 \%$ in water $w / w, 3$ equiv.) was added and stirred at room temperature for another $6 \mathrm{~h}$ and monitored by TLC and/or GC-MS. ${ }^{b}$ Isolated yield based on 2a. ${ }^{c} 60{ }^{\circ} \mathrm{C}$.

物 3a 和 3g $\sim 3 \mathbf{k}($ Entries 7 12). 随后, 对磷叶立德的底 物范围进行了初步探索，结果发现：酮基相连的芳环上， 不论是供电子基还是吸电子基，以及稠环、杂芳环取代 基均可以以较高的收率得到预期产物 $31 \sim 3 q$ (Entries 13 18). 对于吲哚或吡咯取代的酮基稳定磷叶立德, 可能由于吲哚、吡咯基比较活泼, 在标准条件下只能得 到一个复杂的混合物, 不具备制备价值(Entries 19, 20). 对于脂肪酮基稳定的磷叶立德，在标准条件下收率较 低; 但是, 将反应温度升高到 $125{ }^{\circ} \mathrm{C}$ 时, 也可以顺利地 得到预期产物 3t $\sim 3 \mathbf{v}$ (Entries 21 23). 可能是由于 $\alpha$-酮 酯稳定的磷叶立德过于活泼, 在标准反应条件下, 并不 能得到预期产物 $3 \mathrm{w}$ (Entry 24). 上述的合成方法可以很 方便地应用于肉桂醇与酯基或氰基稳定磷叶立德的偶 联反应，只需将反应溶剂更换为 $N, N$-二甲基甲酰胺，将 反应温度提高到 $125{ }^{\circ} \mathrm{C}$, 以中等到较高的收率得到官 能化 1,4-二烯 3x $\sim 3 \mathbf{y}$ (Entries 25，26). 然而, 该方法不 能很好地应用于酰胺基稳定的磷叶立德, 在标准反应条 件下不能得到预期产物 $\mathbf{3 z}$ (Entry 27).

根据相关的实验结果及文献调研 ${ }^{[8 \sim 10]}$, 该反应可能 的机理如 Scheme 2 所示. 首先烯丙基醇在钯催化剂和 硼酸的共同作用下, $\mathrm{C}-\mathrm{O}$ 键活化发生断裂, 生成 $\pi$-烯丙 基钯正离子中间体 5; 磷叶立德 2 作为亲核试剂进攻 $\pi$ 烯丙基钯正离子中间体 $\mathbf{5}$, 进而生成膦盐中间体 
表 2 钯催化下稳定磷叶立德与烯丙基醇的脱水偶联反应

Table 2 Palladium-catalyzed dehydrative allylic cross coupling of stabilized phosphorus ylides with allylic alcohols

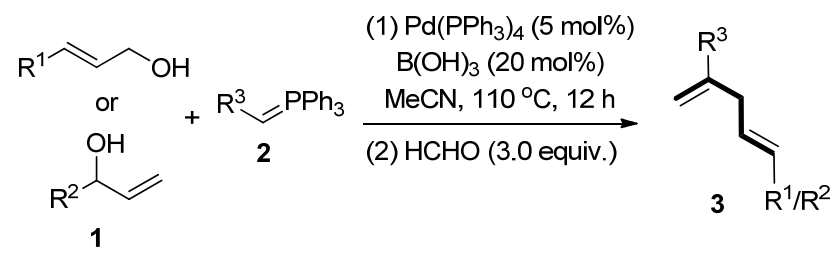

\begin{tabular}{|c|c|c|c|c|c|}
\hline Entry & $\mathrm{y} \quad \mathrm{R}^{1}$ & $\mathrm{R}^{2}$ & $\mathrm{R}^{3}$ & 3 & Yield/\% \\
\hline 1 & $\mathrm{Ph}$ & - & $\mathrm{PhCO}$ & $\mathbf{3 a}$ & 84 \\
\hline 2 & $4-\mathrm{MeOC}_{6} \mathrm{H}_{4}$ & - & $\mathrm{PhCO}$ & $3 \mathbf{b}$ & 95 \\
\hline 3 & $4-\mathrm{NO}_{2} \mathrm{C}_{6} \mathrm{H}_{4}$ & - & $\mathrm{PhCO}$ & $3 \mathbf{c}$ & Trace \\
\hline 4 & $2-\mathrm{MeOC}_{6} \mathrm{H}_{4}$ & - & $\mathrm{PhCO}$ & 3d & 94 \\
\hline 5 & $n-\mathrm{C}_{3} \mathrm{H}_{7}$ & - & $\mathrm{PhCO}$ & $3 \mathbf{e}$ & $61^{c}$ \\
\hline 6 & $\mathrm{H}$ & - & $\mathrm{PhCO}$ & $3 f$ & 78 \\
\hline 7 & - & $\mathrm{Ph}$ & $\mathrm{PhCO}$ & $3 \mathbf{a}$ & 86 \\
\hline 8 & - & $4-\mathrm{ClC}_{6} \mathrm{H}_{4}$ & $\mathrm{PhCO}$ & $3 g$ & 68 \\
\hline 9 & - & 2-Thienyl & $\mathrm{PhCO}$ & $3 \mathbf{h}$ & 80 \\
\hline 10 & - & 1-Naphthyl & $\mathrm{PhCO}$ & $3 \mathbf{i}$ & 75 \\
\hline 11 & - & 2-Naphthyl & $\mathrm{PhCO}$ & $3 \mathbf{j}$ & 80 \\
\hline 12 & - & Et & $\mathrm{PhCO}$ & $3 \mathbf{k}$ & $65^{c}$ \\
\hline 13 & $\mathrm{Ph}$ & - & $4-\mathrm{MeOC}_{6} \mathrm{H}_{4} \mathrm{CO}$ & 31 & 86 \\
\hline 14 & $\mathrm{Ph}$ & - & $4-\mathrm{FC}_{6} \mathrm{H}_{4} \mathrm{CO}$ & $3 m$ & 78 \\
\hline 15 & $\mathrm{Ph}$ & - & $4-\mathrm{ClC}_{6} \mathrm{H}_{4} \mathrm{CO}$ & $3 n$ & 75 \\
\hline 16 & $\mathrm{Ph}$ & - & 2-NaphthylCO & 30 & 84 \\
\hline 17 & $\mathrm{Ph}$ & - & 2-FurylCO & $3 p$ & 73 \\
\hline 18 & $\mathrm{Ph}$ & - & 2-ThienylCO & $3 q$ & 62 \\
\hline 19 & $\mathrm{Ph}$ & - & 3-IndolylCO & $3 \mathbf{r}$ & Complex \\
\hline 20 & $\mathrm{Ph}$ & - & 2-PyrrylCO & $3 \mathrm{~s}$ & Complex \\
\hline 21 & $\mathrm{Ph}$ & - & $t$-BuCO & $3 t$ & $52^{c}$ \\
\hline 22 & $\mathrm{Ph}$ & - & $\mathrm{MeCO}$ & $3 \mathbf{u}$ & $65^{c}$ \\
\hline 23 & $\mathrm{Ph}$ & - & CyclopropylCO & $3 \mathbf{v}$ & $58^{c}$ \\
\hline 24 & $\mathrm{Ph}$ & - & $\mathrm{COCO}_{2} \mathrm{Et}$ & $3 w$ & Complex \\
\hline 25 & $\mathrm{Ph}$ & - & $\mathrm{CO}_{2} \mathrm{Et}$ & $3 x$ & $82^{d}$ \\
\hline 26 & $\mathrm{Ph}$ & - & $\mathrm{CN}$ & $3 \mathbf{y}$ & $64^{d}$ \\
\hline 27 & $\mathrm{Ph}$ & - & $\mathrm{CONH}_{2}$ & $3 z$ & $0^{e}$ \\
\hline
\end{tabular}

${ }^{a}$ See Table 1 Entry 1 for details. ${ }^{b}$ Isolated yield based on 2a. ${ }^{c} 125{ }^{\circ} \mathrm{C} .{ }^{d}$ $\mathrm{N}, \mathrm{N}$-dimethylformamide (DMF) as the solvent. ${ }^{e}$ Ylide was decomposed under the reaction conditions.

6 , 然后转化为新的磷叶立德中间体 7, 最后, 7 与甲醛发 生 Wittig 反应得到最终预期产物 $\mathbf{3}$.

\section{2 结论}

发展了一种钯催化下酮基稳定的磷叶立德与烯丙 基醇一锅法的烯丙化-Wittig 反应. 研究表明, 在 $5 \mathrm{~mol} \%$ 四(三苯基膦)钯和 $20 \mathrm{~mol} \%$ 嗍酸的共催化下，可以以

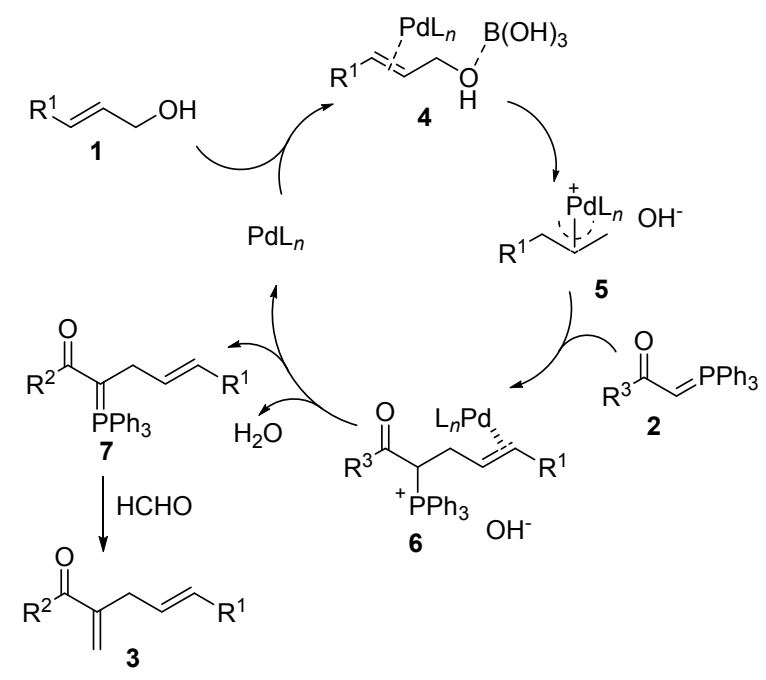

图式 2 可能的反应路径.

Scheme 2 Possible reaction paths

52\% 95\%的收率得到官能化 1,4-二烯化合物. 该方法 还可以进一步拓展到酯基以及氧基稳定的磷叶立德来 合成对应的 1,4-二烯化合物。

\section{3 实验部分}

\section{1 仪器与试剂}

所用化学试剂和溶剂均为商业可得, 没有经过处 理，直接使用. 使用薄层色谱(TLC)和/或气质色谱(GCMS)技术跟踪反应(HSGF254 高效板, 紫外灯检测波长 $254,365 \mathrm{~nm})$. 产物的 ${ }^{1} \mathrm{H}$ NMR, ${ }^{13} \mathrm{C}$ NMR 用 JNM-ECZ600R/ S3 (Jeol, Japan) (600 MHz, $150 \mathrm{MHz}$ )型核磁共振仪测定, $\mathrm{CDCl}_{3}$ 作为気代溶剂. 低分辨质谱 $\mathrm{MS}$ (EI) 用 Agient GC-MS-5890A/5975C Plus spectrometer (EI)测定. 高分 辨质谱使用 LC-TOF (Xevo G2-XS QTof)高分辨质谱仪 以 ESI 电离源测定. 柱层析使用黄海 300 400 目硅胶. 所有试剂均购于试剂公司，未经进一步纯化.

\section{2 实验方法}

3.2.1 钯催化下稳定磷叶立德与烯丙基醇的脱水偶 联反应

氮气保护下，向 $10 \mathrm{~mL}$ 的 Schlenk 反应管内依次加 入肉桂醇 1a (48.2 mg, $0.36 \mathrm{mmol})$, 磷叶立德 2a (114.0 $\mathrm{mg}, 0.30 \mathrm{mmol}$ ), 嗍酸 $(3.7 \mathrm{mg}, 20 \mathrm{~mol} \%$ ), 四(三苯基膦) 钯 $(17.3 \mathrm{mg}, 5 \mathrm{~mol} \%)$ 和乙腈 $(0.5 \mathrm{~mL})$, 加毕置于 $110{ }^{\circ} \mathrm{C}$ 油浴上加热搅拌反应 $12 \mathrm{~h}$. 待反应液冷却后, 加入甲醛 水溶液(质量分数 $37 \%$ in water, $0.068 \mathrm{~mL}, 0.90 \mathrm{mmol}$ ), 室温下继续搅拌反应 $6 \mathrm{~h}$, 柱层析分离 $[V$ (乙酸乙酯)： $V$ (石油醚 $)=0 ： 100 \sim 1 ： 30$ ]得产物 3a.

(E)-1-苯基-4-苯甲酰基-1,4-戊二烯 $(3 a)^{[10]}$ : 无色油. ${ }^{1} \mathrm{H}$ NMR $\left(600 \mathrm{MHz}, \mathrm{CDCl}_{3}\right) \delta: 7.82 \sim 7.76(\mathrm{~m}, 2 \mathrm{H})$, 
$7.57 \sim 7.52(\mathrm{~m}, 1 \mathrm{H}), 7.47 \sim 7.42(\mathrm{~m}, 2 \mathrm{H}), 7.38(\mathrm{~d}, J=7.8$ $\mathrm{Hz}, 2 \mathrm{H}), 7.31(\mathrm{t}, J=7.8 \mathrm{~Hz}, 2 \mathrm{H}), 7.22(\mathrm{t}, J=7.2 \mathrm{~Hz}, 1 \mathrm{H})$, $6.52(\mathrm{~d}, J=15.8 \mathrm{~Hz}, 1 \mathrm{H}), 6.31$ (dt, $J=15.8,7.2 \mathrm{~Hz}, 1 \mathrm{H}$ ), $5.95(\mathrm{~s}, 1 \mathrm{H}), 5.72(\mathrm{~s}, 1 \mathrm{H}), 3.39(\mathrm{~d}, J=7.2 \mathrm{~Hz}, 2 \mathrm{H}) ;{ }^{13} \mathrm{C}$ NMR $\left(150 \mathrm{MHz}, \mathrm{CDCl}_{3}\right) \delta: 197.9,146.6,137.8,137.4$, $132.6,132.4,129.6,128.7,128.6,127.4,127.0,126.7$, 126.3, 35.5; MS (EI) $m / z: 248$.

(E)-1-(4- 甲氧基苯基)-4-苯甲酰基-1,4-戊二烯 (3b) ${ }^{[10]}$ : 无色油. ${ }^{1} \mathrm{H}$ NMR $\left(600 \mathrm{MHz}, \mathrm{CDCl}_{3}\right) \delta: 7.75(\mathrm{dd}$, $J=5.4,3.6 \mathrm{~Hz}, 2 \mathrm{H}), 7.55 \sim 7.51(\mathrm{~m}, 1 \mathrm{H}), 7.45 \sim 7.40(\mathrm{~m}$, $2 \mathrm{H}), 7.32 \sim 7.26(\mathrm{~m}, 2 \mathrm{H}), 6.90 \sim 6.81(\mathrm{~m}, 2 \mathrm{H}), 6.44(\mathrm{~d}, J=$ $15.6 \mathrm{~Hz}, 1 \mathrm{H}), 6.14$ (dt, $J=15.6,7.2 \mathrm{~Hz}, 1 \mathrm{H}), 5.93(\mathrm{~s}, 1 \mathrm{H})$, 5.69 (s, 1H), 3.79 (s, 3H), 3.34 (d, $J=7.2 \mathrm{~Hz}, 2 \mathrm{H}) ;{ }^{13} \mathrm{C}$ NMR $\left(150 \mathrm{MHz}, \mathrm{CDCl}_{3}\right) \delta: 198.0,159.1,146.9,137.8$, $132.4,132.0,130.3,129.6,128.3,127.4,126.8,124.4$, 114.1, 55.4, 35.5; MS (EI) $m / z: 278$.

(E)-1-(2- 甲氧基苯基)-4-苯甲酰基-1,4-戊二烯 (3d) ${ }^{[10]}$ : 无色油. ${ }^{1} \mathrm{H}$ NMR $\left(600 \mathrm{MHz}, \mathrm{CDCl}_{3}\right) \delta$ : 7.84 $7.71(\mathrm{~m}, 2 \mathrm{H}), 7.58 \sim 7.52(\mathrm{~m}, 1 \mathrm{H}), 7.47 \sim 7.42(\mathrm{~m}, 3 \mathrm{H})$, $7.23 \sim 7.16(\mathrm{~m}, 1 \mathrm{H}), 6.94 \sim 6.88(\mathrm{~m}, 3 \mathrm{H}), 6.30(\mathrm{dt}, J=$ 15.6, 7.2 Hz, 1H), 5.95 (s, 1H), $5.70(\mathrm{~s}, 1 \mathrm{H}), 3.83(\mathrm{~s}, 3 \mathrm{H})$, $3.40(\mathrm{~d}, J=6.8 \mathrm{~Hz}, 2 \mathrm{H}) ;{ }^{13} \mathrm{C} \mathrm{NMR}\left(150 \mathrm{MHz}, \mathrm{CDCl}_{3}\right) \delta$ : $198.0,156.5,146.9,137.8,132.3,129.6,128.3,127.2$, 126.7, 126.5, 120.7, 110.9, 55.6, 35.9; MS (EI) $\mathrm{m} / \mathrm{z}: 278$.

(E)-2-苯甲酰基-1,4- 辛二烯 (3e $)^{[10]}$ : 无色油. ${ }^{1} \mathrm{H}$ NMR $\left(600 \mathrm{MHz}, \mathrm{CDCl}_{3}\right) \delta: 7.73(\mathrm{~d}, J=7.8 \mathrm{~Hz}, 2 \mathrm{H})$, $7.55 \sim 7.51(\mathrm{~m}, 1 \mathrm{H}), 7.45 \sim 7.41(\mathrm{~m}, 2 \mathrm{H}), 5.84(\mathrm{~s}, 1 \mathrm{H})$, $5.61(\mathrm{~s}, 1 \mathrm{H}), 5.57 \sim 5.37(\mathrm{~m}, 2 \mathrm{H}), 3.14(\mathrm{~d}, J=6.6 \mathrm{~Hz}, 2 \mathrm{H})$, $2.06 \sim 1.89(\mathrm{~m}, 2 \mathrm{H}), 1.43 \sim 1.35(\mathrm{~m}, 2 \mathrm{H}), 0.86(\mathrm{t}, J=7.2$ $\mathrm{Hz}, 3 \mathrm{H}) ;{ }^{13} \mathrm{C}$ NMR $\left(150 \mathrm{MHz}, \mathrm{CDCl}_{3}\right) \delta: 198.2,147.4$, 137.9, 133.6, 132.2, 129.6, 128.3, 126.2, 125.9, 35.2, 34.7, 22.6, 13.7; MS (EI) $m / z: 214$.

(E)-2-苯甲酰基-1,4-戊二烯(3f ${ }^{[12]}$ : 无色油. ${ }^{1} \mathrm{H}$ NMR $\left(600 \mathrm{MHz}, \mathrm{CDCl}_{3}\right) \delta: 7.75 \sim 7.64(\mathrm{~m}, 2 \mathrm{H}), 7.52 \sim 7.40(\mathrm{~m}$, $1 \mathrm{H}), 7.35(\mathrm{dd}, J=10.8,4.8 \mathrm{~Hz}, 2 \mathrm{H}), 5.92 \sim 5.73(\mathrm{~m}, 2 \mathrm{H})$, 5.59 (s, $1 \mathrm{H}), 5.12 \sim 4.99(\mathrm{~m}, 2 \mathrm{H}), 3.15(\mathrm{~d}, J=6.6 \mathrm{~Hz}, 2 \mathrm{H})$; ${ }^{13} \mathrm{C} \mathrm{NMR}\left(150 \mathrm{MHz}, \mathrm{CDCl}_{3}\right) \delta: 196.7,145.4,136.7,133.9$, 131.2, 128.5, 127.2, 125.4, 116.1, 35.2; MS (EI) $m / z$ : 172.

(E)-1-(4-氯苯基)-4-苯甲酰基-1,4-戊二烯(3g): 无色 固体. m.p. $70 \sim 72{ }^{\circ} \mathrm{C}$ (文献值 ${ }^{[10]} 72 \sim 73{ }^{\circ} \mathrm{C}$ ); ${ }^{1} \mathrm{H}$ NMR $\left(600 \mathrm{MHz}, \mathrm{CDCl}_{3}\right) \delta: 7.76(\mathrm{dd}, J=7.8,1.2 \mathrm{~Hz}, 2 \mathrm{H})$, $7.58 \sim 7.40(\mathrm{~m}, 3 \mathrm{H}), 7.38 \sim 7.21(\mathrm{~m}, 4 \mathrm{H}), 6.45(\mathrm{~d}, J=15.6$ $\mathrm{Hz}, 1 \mathrm{H}), 6.27$ (dt, $J=15.6,7.2 \mathrm{~Hz}, 1 \mathrm{H}), 5.94(\mathrm{~s}, 1 \mathrm{H}), 5.72$ (s, 1H), 3.37 (d, $J=6.6 \mathrm{~Hz}, 2 \mathrm{H}) ;{ }^{13} \mathrm{C}$ NMR $(150 \mathrm{MHz}$, $\left.\mathrm{CDCl}_{3}\right) \delta: 197.7,146.4,137.7,135.9,132.9,132.4,131.3$,
129.6, 128.8, 128.4, 127.5, 127.1, 35.6; MS (EI) $m / z: 282$

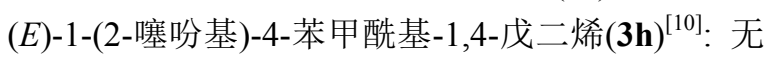
色油. ${ }^{1} \mathrm{H}$ NMR (600 MHz, $\mathrm{CDCl}_{3}$ ) $\delta: 7.75$ (dd, $J=5.4,3.6$ $\mathrm{Hz}, 2 \mathrm{H}) 7.55 \sim 7.50(\mathrm{~m}, 1 \mathrm{H}), 7.43(\mathrm{t}, J=7.8 \mathrm{~Hz}, 2 \mathrm{H}), 7.10$ (d, $J=5.4 \mathrm{~Hz}, 1 \mathrm{H}), 6.97 \sim 6.84(\mathrm{~m}, 2 \mathrm{H}), 6.63(\mathrm{~d}, J=15.6$ $\mathrm{Hz}, 1 \mathrm{H}), 6.13$ (dt, $J=15.6,7.2 \mathrm{~Hz}, 1 \mathrm{H}), 5.94$ (s, 1H), 5.71 (s, 1H), $3.34(\mathrm{~d}, J=7.2 \mathrm{~Hz}, 2 \mathrm{H}) ;{ }^{13} \mathrm{C}$ NMR $(150 \mathrm{MHz}$, $\left.\mathrm{CDCl}_{3}\right) \delta: 197.7,146.3,142.5,137.7,132.4,129.6,128.3$, 127.4, 127.1, 126.4, 126.2, 125.1, 123.8, 35.3; MS (EI) $m / z: 254$.

(E)-1-(1-菜基)-4-苯甲酰基-1,4-戊二烯(3i): 无色固 体, m.p. $65 \sim 66{ }^{\circ} \mathrm{C}$ (文献值 ${ }^{[10]}$ m.p. $68 \sim 69{ }^{\circ} \mathrm{C}$ ); ${ }^{1} \mathrm{H}$ NMR $\left(600 \mathrm{MHz}, \mathrm{CDCl}_{3}\right) \delta: 8.10 \sim 8.05(\mathrm{~m}, 1 \mathrm{H}), 7.83 \sim 7.77(\mathrm{~m}$, $3 \mathrm{H}), 7.73(\mathrm{~d}, J=8.2 \mathrm{~Hz}, 1 \mathrm{H}), 7.60 \sim 7.40(\mathrm{~m}, 7 \mathrm{H}), 7.24(\mathrm{~d}$, $J=15.6 \mathrm{~Hz}, 1 \mathrm{H}), 6.29(\mathrm{dt}, J=15.6,7.2 \mathrm{~Hz}, 1 \mathrm{H}), 5.98(\mathrm{~s}$, $1 \mathrm{H}), 5.73(\mathrm{~s}, 1 \mathrm{H}), 3.49(\mathrm{~d}, J=6.6 \mathrm{~Hz}, 2 \mathrm{H}) ;{ }^{13} \mathrm{C}$ NMR $(150$ $\left.\mathrm{MHz}, \mathrm{CDCl}_{3}\right) \delta: 197.8,171.2,146.6,137.7,135.2,133.7$, $132.4,131.2,129.9,129.6,128.5,128.3,127.7,126.8$, 126.0, 125.8, 125.7, 123.9, 123.8, 35.2; MS (EI) $\mathrm{m} / \mathrm{z}: 298$.

(E)-1-(2-苶基)-4-苯甲酰基-1,4-戊二烯 $(\mathbf{3} \mathbf{j})$ : 无色固 体, m.p. 73 74 ${ }^{\circ} \mathrm{C}$ (文献值 ${ }^{[10]}$ m.p. $76 \sim 77{ }^{\circ} \mathrm{C}$ ); ${ }^{1} \mathrm{H}$ NMR $\left(600 \mathrm{MHz}, \mathrm{CDCl}_{3}\right) \delta: 8.10(\mathrm{~d}, J=7.8 \mathrm{~Hz}, 1 \mathrm{H}), 7.86 \sim 7.80$ (m, 3H), $7.77(\mathrm{~d}, J=7.8 \mathrm{~Hz}), 7.59 \sim 7.40(\mathrm{~m}, 7 \mathrm{H}), 7.27(\mathrm{~d}$, $J=15.6 \mathrm{~Hz}, 1 \mathrm{H}), 6.48 \sim 6.19(\mathrm{dt}, J=15.6,7.2 \mathrm{~Hz}, 1 \mathrm{H})$, $6.02(\mathrm{~s}, 1 \mathrm{H}), 5.76(\mathrm{~s}, 1 \mathrm{H}), 3.52(\mathrm{~d}, J=7.2 \mathrm{~Hz}, 2 \mathrm{H}) ;{ }^{13} \mathrm{C}$ NMR $\left(150 \mathrm{MHz}, \mathrm{CDCl}_{3}\right) \delta: 197.9,146.7,137.8,135.3$, $133.7,132.4,131.2,129.9,129.7,128.6,128.4,127.8$, 127.0, 126.1, 125.9, 125.8, 124.0, 123.9, 35.9; MS (EI) $\mathrm{m} / \mathrm{z}: 298$.

(E)-2-苯甲酰基-1,4-庚二烯 (3k $)^{[10]}$ : 无色油. ${ }^{1} \mathrm{H}$ NMR (600 MHz, $\left.\mathrm{CDCl}_{3}\right) \delta: 7.74(\mathrm{~d}, J=7.8 \mathrm{~Hz}, 2 \mathrm{H})$, $7.59 \sim 7.47(\mathrm{~m}, 1 \mathrm{H}), 7.42(\mathrm{t}, J=7.8 \mathrm{~Hz}, 2 \mathrm{H}), 5.84(\mathrm{~d}, J=$ $1.0 \mathrm{~Hz}, 1 \mathrm{H}), 5.61(\mathrm{~s}, 1 \mathrm{H}), 5.60 \sim 5.55(\mathrm{~m}, 1 \mathrm{H}), 5.48 \sim 5.41$ (m, $1 \mathrm{H}), 3.13(\mathrm{~d}, J=6.5 \mathrm{~Hz}, 2 \mathrm{H}), 2.06 \sim 2.00(\mathrm{~m}, 2 \mathrm{H})$, $0.96(\mathrm{t}, J=7.2 \mathrm{~Hz}, 3 \mathrm{H}) ;{ }^{13} \mathrm{C} \mathrm{NMR}\left(150 \mathrm{MHz}, \mathrm{CDCl}_{3}\right) \delta$ : $198.2,147.4,137.9,135.3,132.2,129.6,128.3,126.0$, 125.1, 35.1, 25.7, 13.9; MS (EI) m/z: 200.

(E)-1-苯基-4-(4-甲氧基苯甲酰基)-1,4-戊二烯(3I): 无色固体, m.p. $38 \sim 39{ }^{\circ} \mathrm{C}\left(\right.$ 文献值 ${ }^{[10]}$ m.p. $38 \sim 39{ }^{\circ} \mathrm{C}$ ); ${ }^{1} \mathrm{H}$ NMR $\left(600 \mathrm{MHz}, \mathrm{CDCl}_{3}\right) \delta: 7.82(\mathrm{~d}, J=9.0 \mathrm{~Hz}, 2 \mathrm{H})$, $7.34(\mathrm{~d}, J=7.8 \mathrm{~Hz}, 2 \mathrm{H}), 7.28(\mathrm{t}, J=7.8 \mathrm{~Hz}, 2 \mathrm{H}), 7.19$ (t, $J=7.8 \mathrm{~Hz}, 1 \mathrm{H}), 6.97 \sim 6.91(\mathrm{~m}, 2 \mathrm{H}), 6.49(\mathrm{~d}, J=15.6 \mathrm{~Hz}$, $1 \mathrm{H}), 6.27$ (dt, $J=15.6,7.2 \mathrm{~Hz}, 1 \mathrm{H}), 5.82(\mathrm{~s}, 1 \mathrm{H}), 5.61$ (s, $1 \mathrm{H}), 3.84$ (s, 3H), 3.36 (d, $J=7.2 \mathrm{~Hz}, 2 \mathrm{H}) ;{ }^{13} \mathrm{C}$ NMR $(150$ $\left.\mathrm{MHz}, \mathrm{CDCl}_{3}\right) \delta: 196.5,163.2,146.8,137.4,132.4,132.0$, 
$130.1,128.5,127.3,126.7,126.2,124.5,113.6,55.5,35.9$; MS (EI) $m / z: 278$.

(E)-1-苯基-4-(4-氟苯甲酰基)-1,4-戊二烯 $(3 \mathbf{m})^{[10]}$ : 无色油. ${ }^{1} \mathrm{H} \mathrm{NMR}\left(600 \mathrm{MHz}, \mathrm{CDCl}_{3}\right) \delta: 7.84 \sim 7.79(\mathrm{~m}$, 2H), 7.36 (d, $J=7.2 \mathrm{~Hz}, 2 \mathrm{H}), 7.30$ (t, $J=7.8 \mathrm{~Hz}, 2 \mathrm{H}), 7.22$ $(\mathrm{d}, J=7.2 \mathrm{~Hz}, 1 \mathrm{H}), 7.14 \sim 7.08(\mathrm{~m}, 2 \mathrm{H}), 6.50(\mathrm{~d}, J=15.6$ $\mathrm{Hz}, 1 \mathrm{H}), 6.28$ (dt, $J=15.6,7.2 \mathrm{~Hz}, 1 \mathrm{H}), 5.93$ (s, 1H), 5.67 $(\mathrm{s}, 1 \mathrm{H}), 3.36(\mathrm{~d}, J=7.2 \mathrm{~Hz}, 2 \mathrm{H}) ;{ }^{13} \mathrm{C} \mathrm{NMR}(150 \mathrm{MHz}$, $\left.\mathrm{CDCl}_{3}\right) \delta: 196.4,165.4(\mathrm{~d}, J=253.7 \mathrm{~Hz}), 146.6,137.3$, 133.8, 132.7, 132.2 (d, $J=9.3 \mathrm{~Hz}), 128.6,127.4,126.4$ (d, $J=10.2 \mathrm{~Hz}), 126.3,115.5$ (d, $J=21.8 \mathrm{~Hz}), 35.6$; MS (EI) $m / z: 266$.

$(E)-1$-苯基-4-(4-氯苯甲酰基)-1,4-戊二烯(3n): 无色 固体, m.p. 52 53 ${ }^{\circ} \mathrm{C}$ (文献值 ${ }^{[10]}$ m.p. $51 \sim 52 ~{ }^{\circ} \mathrm{C}$ ); ${ }^{1} \mathrm{H}$ NMR (600 MHz, $\left.\mathrm{CDCl}_{3}\right) \delta: 7.72(\mathrm{dd}, J=8.4,2.4 \mathrm{~Hz}, 2 \mathrm{H})$, $7.45 \sim 7.41(\mathrm{~m}, 2 \mathrm{H}), 7.36(\mathrm{~d}, J=7.2 \mathrm{~Hz}, 2 \mathrm{H}), 7.33 \sim 7.28$ (m, 2H), 7.22 (t, $J=7.2 \mathrm{~Hz}, 1 \mathrm{H}), 6.51(\mathrm{~d}, J=15.6 \mathrm{~Hz}, 1 \mathrm{H})$, $6.28(\mathrm{dt}, J=15.6,7.2 \mathrm{~Hz}, 1 \mathrm{H}), 5.95$ (s, 1H), 5.68 (s, 1H), $3.37(\mathrm{~d}, J=7.2 \mathrm{~Hz}, 2 \mathrm{H}) ;{ }^{13} \mathrm{C} \mathrm{NMR}\left(150 \mathrm{MHz}, \mathrm{CDCl}_{3}\right) \delta$ : $196.6,146.5,138.8,137.3,136.0,132.8,131.0,128.7$, 128.6, 127.4, 127.0, 126.3, 126.2, 35.5; MS (EI) $m / z: 282$.

(E)-1-苯基-4-(2-菜甲酰基)-1,4-戊二烯(3o): 无色固 体, m.p. 53 54 ${ }^{\circ} \mathrm{C}$ (文献值 ${ }^{[10]}$ m.p. 56 57 $\left.{ }^{\circ} \mathrm{C}\right) ;{ }^{1} \mathrm{H}$ NMR $\left(600 \mathrm{MHz}, \mathrm{CDCl}_{3}\right) \delta: 8.30(\mathrm{~s}, 1 \mathrm{H}), 7.96 \sim 7.86(\mathrm{~m}, 4 \mathrm{H})$, $7.62 \sim 7.53(\mathrm{~m}, 2 \mathrm{H}), 7.44 \sim 7.38(\mathrm{~m}, 2 \mathrm{H}), 7.34 \sim 7.28(\mathrm{~m}$, 2H), $7.25 \sim 7.17(\mathrm{~m}, 1 \mathrm{H}), 6.57(\mathrm{~d}, J=15.6 \mathrm{~Hz}, 1 \mathrm{H}), 6.37$ $(\mathrm{dt}, J=15.6,7.2 \mathrm{~Hz}, 1 \mathrm{H}), 6.00(\mathrm{~s}, 1 \mathrm{H}), 5.79$ (s, 1H), 3.46 $(\mathrm{d}, J=7.2 \mathrm{~Hz}, 2 \mathrm{H}) ;{ }^{13} \mathrm{C} \mathrm{NMR}\left(150 \mathrm{MHz}, \mathrm{CDCl}_{3}\right) \delta: 197.9$, $146.8,137.4,135.3,135.0,132.7,132.4,131.3,129.5$, $128.7,128.4,128.3,127.9,127.4,126.9,126.8,126.7$, 126.3, 125.6, 35.8; MS (EI) $m / z: 298$.

(E)-1-苯基-4-(2-呋喃甲酰基)-1,4-戊二烯(3p $)^{[10]}$ : 无 色油. ${ }^{1} \mathrm{H}$ NMR (600 MHz, $\left.\mathrm{CDCl}_{3}\right) \delta: 7.62(\mathrm{~s}, 1 \mathrm{H}), 7.37 \sim$ $7.24(\mathrm{~m}, 4 \mathrm{H}), 7.20(\mathrm{t}, J=7.2 \mathrm{~Hz}, 1 \mathrm{H}), 7.21 \sim 7.12(\mathrm{~m}, 1 \mathrm{H})$, $6.50(\mathrm{dd}, J=3.6,1.8 \mathrm{~Hz}, 1 \mathrm{H}), 6.45(\mathrm{~d}, J=15.6 \mathrm{~Hz}, 1 \mathrm{H})$, $6.22(\mathrm{dt}, J=15.6,7.2 \mathrm{~Hz}, 1 \mathrm{H}), 6.03(\mathrm{~s}, 1 \mathrm{H}), 5.82(\mathrm{~s}, 1 \mathrm{H})$, $3.32(\mathrm{~d}, J=7.2 \mathrm{~Hz}, 2 \mathrm{H}) ;{ }^{13} \mathrm{C} \mathrm{NMR}\left(150 \mathrm{MHz}, \mathrm{CDCl}_{3}\right) \delta$ : $183.5,151.8,147.0,146.1,137.1,132.4,128.4,127.1$, 126.2, 126.0, 124.6, 119.9, 111.9, 35.2; MS (EI) $\mathrm{m} / \mathrm{z}: 238$.

(E)-1-苯基-4-(2-噻吩甲酰基)-1,4-戊二烯 $(\mathbf{3 p})^{[10]}$ : 无 色油. ${ }^{1} \mathrm{H} \mathrm{NMR}\left(400 \mathrm{MHz}, \mathrm{CDCl}_{3}\right) \delta: 7.72 \sim 7.64(\mathrm{~m}, 2 \mathrm{H})$, $7.37 \sim 7.19(\mathrm{~m}, 5 \mathrm{H}), 7.13 \sim 7.10(\mathrm{~m}, 1 \mathrm{H}), 6.48(\mathrm{~d}, J=15.6$ $\mathrm{Hz}, 1 \mathrm{H}), 6.27$ (dt, $J=15.6,7.2 \mathrm{~Hz}, 1 \mathrm{H}), 5.89$ (d, $J=0.6$ $\mathrm{Hz}, 1 \mathrm{H}), 5.81(\mathrm{~d}, J=0.6 \mathrm{~Hz}, 1 \mathrm{H}), 3.36(\mathrm{dd}, J=7.2,0.6 \mathrm{~Hz}$, $2 \mathrm{H}) ;{ }^{13} \mathrm{C} \mathrm{NMR}\left(100 \mathrm{MHz}, \mathrm{CDCl}_{3}\right) \delta: 189.1,147.0,143.4$,
$137.3,134.1,133.9,132.6,128.5,127.9,127.3,126.3$, 126.2, 123.9, 35.8; MS (EI) $m / z: 254$.

$(E)-1$-苯基-4-(叔丁基甲酰基)-1,4-戊二烯(3t) ${ }^{[10]}$ : 无 色油. ${ }^{1} \mathrm{H}$ NMR $\left(600 \mathrm{MHz}, \mathrm{CDCl}_{3}\right) \delta: 7.35(\mathrm{~d}, J=7.2 \mathrm{~Hz}$, $2 \mathrm{H}), 7.30(\mathrm{t}, J=7.2 \mathrm{~Hz}, 2 \mathrm{H}), 7.23 \sim 7.17(\mathrm{~m}, 1 \mathrm{H}), 6.42(\mathrm{~d}$, $J=15.6 \mathrm{~Hz}, 1 \mathrm{H}), 6.18(\mathrm{dt}, J=15.6,7.2 \mathrm{~Hz}, 1 \mathrm{H}), 5.54(\mathrm{~s}$, 1H), 5.49 (d, $J=1.2 \mathrm{~Hz}, 1 \mathrm{H}), 3.14$ (dd, $J=7.2,1.2 \mathrm{~Hz}$, $2 \mathrm{H}), 1.25$ (s, 9H); ${ }^{13} \mathrm{C}$ NMR (150 MHz, $\left.\mathrm{CDCl}_{3}\right) \delta: 211.0$, $146.9,137.4,132.5,128.6,127.4,126.7,126.2,118.6$, 44.2, 37.6, 27.9; MS (EI) $m / z: 228$.

(E)-1-苯基-4-(乙酰基)-1,4-戊二烯(3u) ${ }^{[10]}$ : 无色油. ${ }^{1} \mathrm{H}$ NMR $\left(600 \mathrm{MHz}, \mathrm{CDCl}_{3}\right) \delta: 7.40 \sim 7.31(\mathrm{~m}), 7.31 \sim$ $7.23(\mathrm{~m}), 7.20$ (t, $J=7.4 \mathrm{~Hz}), 6.42(\mathrm{~d}, J=15.8 \mathrm{~Hz}), 6.21$ (dt, $J=15.7,7.0 \mathrm{~Hz}), 6.08$ (s), 5.85 (d, $J=1.2 \mathrm{~Hz}), 3.17$ (d, $J=6.9 \mathrm{~Hz}), 2.36(\mathrm{~s}) ;{ }^{13} \mathrm{C} \mathrm{NMR}\left(150 \mathrm{MHz}, \mathrm{CDCl}_{3}\right) \delta$ : $199.4,147.7,137.4,132.1,128.6,127.3,127.2,126.2$, 126.1, 34.0, 26.0; MS (EI) $m / z: 186$.

(E)-1-苯基-4-(环丙甲酰基)-1,4-戊二烯(3v)：无色 油. ${ }^{1} \mathrm{H}$ NMR $\left(600 \mathrm{MHz}, \mathrm{CDCl}_{3}\right) \delta: 7.47 \sim 7.16(\mathrm{~m}, 5 \mathrm{H})$, $6.43(\mathrm{~d}, J=15.8 \mathrm{~Hz}, 1 \mathrm{H}), 6.29 \sim 6.15(\mathrm{~m}, 2 \mathrm{H}), 5.83(\mathrm{~s}$, $1 \mathrm{H}), 3.21(\mathrm{~d}, J=7.2 \mathrm{~Hz}, 2 \mathrm{H}), 2.54 \sim 2.39(\mathrm{~m}, 1 \mathrm{H}), 1.11 \sim$ $1.07(\mathrm{~m}, 2 \mathrm{H}), 0.95 \sim 0.90(\mathrm{~m}, 3 \mathrm{H}) ;{ }^{13} \mathrm{C} \mathrm{NMR}(150 \mathrm{MHz}$, $\left.\mathrm{CDCl}_{3}\right) \delta: 201.42$ (s), 148.16 (s), 137.50 (s), 132.08 (s), 128.59 (s), 127.28 (d, J=11.8 Hz), 126.19 (s), 124.50 (s), 34.53 (s), 16.51 (s), 11.34 (s); MS (EI) m/z: 212. HRMS (ESI) calcd for $\mathrm{C}_{15} \mathrm{H}_{17} \mathrm{O}(\mathrm{M}+\mathrm{H})^{+}$213.1279, found 213.1295

2-肉桂基-丙烯酸乙酯 $(\mathbf{3 x})^{[8 \mathrm{a}]}$ : 无色油. ${ }^{1} \mathrm{H}$ NMR $\left(600 \mathrm{MHz}, \mathrm{CDCl}_{3}\right) \delta: 7.37 \sim 7.34(\mathrm{~m}, 2 \mathrm{H}), 7.31 \sim 7.27(\mathrm{~m}$, $2 \mathrm{H}), 7.22 \sim 7.18(\mathrm{~m}, 1 \mathrm{H}), 6.44(\mathrm{~d}, J=15.6 \mathrm{~Hz}, 1 \mathrm{H}), 6.27 \sim$ $6.21(\mathrm{~m}, 2 \mathrm{H}), 5.61(\mathrm{~d}, J=1.2 \mathrm{~Hz}), 4.22(\mathrm{q}, J=7.2 \mathrm{~Hz})$, $3.20(\mathrm{~d}, J=7.2 \mathrm{~Hz}), 1.30(\mathrm{t}, J=7.2 \mathrm{~Hz}) ;{ }^{13} \mathrm{C}$ NMR $(150$ $\left.\mathrm{MHz}, \mathrm{CDCl}_{3}\right) \delta: 171.2,139.5,137.4,132.1,128.6,127.3$, 126.9, 125.5, 60.8, 35.2, 14.3; MS (EI) $m / z: 216$.

2-肉桂基-丙烯腈 $(3 y))^{[13]}$ : 无色油. ${ }^{1} \mathrm{H}$ NMR (600 $\left.\mathrm{MHz}, \mathrm{CDCl}_{3}\right) \delta: 7.40 \sim 7.20(\mathrm{~m}, 5 \mathrm{H}), 6.53(\mathrm{~d}, J=15.6 \mathrm{~Hz}$, $1 \mathrm{H}), 6.16$ (dt, $J=15.6,6.6 \mathrm{~Hz}, 1 \mathrm{H}), 5.92$ (s, 1H), 5.80 (d, $J=1.8 \mathrm{~Hz}, 1 \mathrm{H}), 3.18 \sim 3.12(\mathrm{~m}, 2 \mathrm{H}) ;{ }^{13} \mathrm{C} \mathrm{NMR}(150 \mathrm{MHz}$, $\left.\mathrm{CDCl}_{3}\right) \delta: 136.6,134.2,130.9,128.6,127.8,126.4,123.2$, 121.8, 118.5, 37.7; MS (EI) $m / z: 169$.

辅助材料(Supporting Information) 所有产物的 ${ }^{1} \mathrm{H}$ $\mathrm{NMR}$ 和 ${ }^{13} \mathrm{C} \mathrm{NMR}$ 谱图. 对酯基稳定磷叶立德的反应条 件进一步考察及结果. 这些材料可以免费从本刊网站 (http://sioc-journal.cn/)上下载. 


\section{References}

[1] (a) Jie, M. S. F. L. K.; Pasha, M. K.; Syed-Rahmatulla, M. S. K. Nat. Prod. Rep. 1997, 14, 163.

(b) Fürstner, A.; Nevado, C.; Waser, M.; Tremblay, M.; Chevrier, C.; Teplý, F.; Aïssa, C.; Moulin, E.; Müller, O. J. Am. Chem. Soc. 2007, 129, 9150.

(c) Wilson, M. C.; Nam, S.-J.; Gulder, T. A. M.; Kauffman, C. A.; Jensen, P. R.; Fenical, W.; Moore, B. S. J. Am. Chem. Soc. 2011, 133, 1971.

[2] (a) Macklin, T. K.; Micalizio, G. C. Nat. Chem. 2010, 2, 638.

(b) Sharma, R. K.; RajanBabu, T. V. J. Am. Chem. Soc. 2010, 132, 3295 .

(c) Trost, B. M.; Luan, X. J. Am. Chem. Soc. 2011, 133, 1706.

(d) McCammant, M. S.; Liao, L.; Sigman, M. S. J. Am. Chem. Soc. 2013, 135, 4167.

(e) Jin, W.; Yang, Q.; Wu, P.; Chen, J.; Yu, Z. Adv. Synth. Catal. 2014, 360, 2097.

[3] (a) Miyaura, N.; Yano, T.; Suzuki, A. Tetrahedron Lett. 1980, 21, 2865.

(b) Kabalka, G. W.; Al-Masum, M. Org. Lett. 2006, 8, 11 .

(c) Lee, Y.; Akiyama, K.; Gillingham, D. G.; Brown, M. K.; Hoveyda, A. H. J. Am. Chem. Soc. 2008, 130, 446.

(d) Akiyama, K.; Gao, F.; Hoveyda, A. H. Angew. Chem., Int. Ed. 2010, 49,419 .

(e) Gao, F.; Lee, K. P.; McGrath, Y.; Hoveyda, A. H. J. Am. Chem. Soc. 2010, 132, 14315.

(f) Gao, F.; Carr, J. L.; Hoveyda, A. H. Angew. Chem., Int. Ed. 2012, 51, 6613.

(g) Huang, Y.; Fañanás-Mastral, M.; Minnaard, A. J.; Feringa, B. L. Chem. Commun. 2013, 49, 3309.

(h) Hamilton, J. Y.; Sarlah, D.; Carreira, E. M. J. Am. Chem. Soc. 2013, 135, 994.

(i) Gao, F.; Carr, J. L.; Hoveyda, A. H. J. Am. Chem. Soc. 2014, 136, 2149.

(j) Sidera, M.; Fletcher, S. P. Chem. Commun. 2015, 51, 5044.

(k) Zhurkin, F. E.; Hu, X. J. Org. Chem. 2016, 81, 5795.

(1) Yang, B.; Wang, Z.-X. J. Org. Chem. 2017, 82, 4542.

[4] (a) Cornella, J.; Zarate, C.; Martin, R. Chem. Soc. Rev. 2014, 43, 8081.

(b) Matsubara, R.; Jamison, T. F. J. Am. Chem. Soc. 2010, 132, 6880 .

(c) Matsubara, R.; Jamison, T. F. Chem. Asian J. 2011, 6, 1860.

(d) Ye, K.-Y.; He, H.; Liu, W.-B.; Dai, L.-X.; Helmchen, G.; You, S.-L. J. Am. Chem. Soc. 2011, 133, 19006.

(e) Hamilton, J. Y.; Sarlah, D.; Carreira, E. M. J. Am. Chem. Soc. 2014, 13 6, 2006.

(f) Gumrukcu, Y.; de Bruin, B.; Reek, J. N. H. Chem.-Eur. J. 2014, 20, 10905 .

[5] (a) Thadani, A. N.; Rawal, V. H. Org. Lett. 2002, 4, 4317.

(b) Chen, X.; Chen, D.; Lu, Z.; Kong, L.; Zhu, G.-G. J. Org. Chem.
2011, 76, 6338 .

(c) Wen, Y.; Jiang, H.-F. Tetrahedron Lett. 2013, 54, 4034.

(d) Todd, D. P.; Thompson, B. B.; Nett, A. J.; Montgomery, J. J. Am. Chem. Soc. 2015, 137, 12788

(e) Mateos, J.; Rivera-Chao, E.; Fañanás-Mastral, M. ACS Catal. 2017, 7, 5340 .

[6] For rare examples for terminal skipped dienes synthesis, see: (a) Basavaiah, D.; Kumaragurubaran, N.; Sharada, D. S. Tetrahedron Lett. 2001, 42, 85 .

(b) Basavaiah, D.; Sharada, D. S.; Kumaragurubaran, N.; Reddy, R. M. J. Org. Chem. 2002, 67, 7135 .

(c) Li, Y.-Q.; Wang, H.-J.; Huang, Z.-Z. J. Org. Chem. 2016, 81, 4429.

[7] For reviews, see: (a) Edmonds, M.; Abell, A. In Modern Carbonyl Olefination, Ed.: Takeda, T., Wiley-VCH, Weinheim, Germany, 2004, pp. 1 17.

(b) Ju, Y. In Modern Organic Reactions, Vol. 3, Eds.: Hu, Y.-F.; Lin, G.-Q., Chemical Industry Press, Beijing, 2008, pp. 413 460 (in Chinese).

(巨勇, 现代有机反应, 主编: 胡跃飞, 林国强, 第 3 卷, 化学工 业出版社, 北京, 2008, pp. 413 460.)

(c) Gu, Y.; Tian, S.-K. Top. Curr. Chem. 2012, 327, 197.

[8] (a) Liu, W.-B.; He, H.; Dai, L.-X.; You, S.-L. Chem.-Eur. J. 2010, 16,7376 .

(b) Ma, X.-T.; Wang, Y.; Dai, R.-H.; Liu, C.-R.; Tian, S.-K. J. Org. Chem. 2013, 78, 11071.

[9] For reviews, see: (a) Bandini, M. Angew. Chem., Int. Ed. 2011, 50, 994.

(b) Sundararaju, B.; Achard, M.; Bruneau, C. Chem. Soc. Rev. 2012, $41,4467$.

(c) Butta, N. A.; Zhang, W. Chem. Soc. Rev. 2015, 44, 7929.

(d) Ferraccioli, R.; Pignataro, L. Curr. Org. Chem. 2015, 19, 106. For selected recent examples: (e) Shen, D.; Chen, Q.; Yan, P.; Zeng, X.; Zhong, G. Angew. Chem., Int. Ed. 2017, 129, 3290.

(f) Wu, F.-P.; Peng, J.-B.; Fu, L.-Y.; Qi, X.; Wu, X.-F. Org. Lett 2017, 19, 5474.

(g) Su, Y.-L.; Han, Z.-Y.; Li, Y.-H.; Gong, L.-Z. ACS Catal. 2017, 7,7917

(h) Jia, X.-G.; Guo, P.; Duan, J.; Shu, X.-Z. Chem. Sci. 2018, 9, 640.

[10] Ma, X.; Yu, J.; Han, C.; Zhou, Q.; Ren, M.; Li, L.; Tang, L. Adv. Synth. Catal. 2019, https://doi.org/10.1002/adsc.201801266.

[11] (a) Ma, X.-T.; Dai, R.-H.; Zhang, J.; Gu, Y.; Tian, S.-K. Adv. Synth. Catal. 2014, 356, 2984.

(b) Ma, X.; Yu, L.; Su, C.; Yang, Y.; Li, H.; Xu, Q. Adv. Synth. Catal. 2017, 359, 1649.

(c) Ma, X.; Xu, Q.; Li, H.; Su, C.; Yu, L.; Zhang, X.; Cao, H.; Han, L.-B. Green Chem. 2018, 20, 3408.

(d) Ma, X.; Su, C.; Xu, Q. Top. Curr. Chem. 2016, 374, 27.

[12] Kaszynski, P.; Friedli, A. C.; Michl, J. J. Am. Chem. Soc. 1992,114, 601.

[13] Liu, H.-J.; Wynn, H. Tetrahedron Lett. 1982, 23, 3151. 